\title{
AN INVARIANT SUBSPACE THEOREM
}

\section{JOHN DAUGHTRY}

ABSTRACT. If $A Y-Y A$ has rank one for some compact $Y$, then $A$ has a nontrivial invariant subspace.

Let $X$ be a complex Banach space and $\mathcal{B}(X)$ the set of bounded linear operators on $\mathcal{X}$. Lomonosov has proved that if $A$ and $Y$ belong to $B(\mathcal{C})$ with $Y$ compact and $A Y-Y A=0$, then $A$ has an invariant subspace [1]. We have obtained an extension of this result.

Theorem. If $A Y-Y A$ has rank one for some compact $Y$, then $A$ has a nontrivial invariant subspace.

This result contrasts with the fact that if $A$ does not commute with a trace class operator then $\{A Y-Y A \mid Y$ is compact $\}$ is uniformly dense in the compacts [4].

The proof of the theorem requires a lemma which may be attributed to David Luenberger although it is incorrectly stated in his paper [2]. The author is grateful to J. P. Williams who pointed out the correct version of the lemma and counterexamples to Luenberger's stronger version and partial converse.

Lemma (Luenberger). Suppose

$$
T A-B T=C
$$

has rank one for $T, A$, and $B$ in $B(X)$. If the largest $A$-invariant subspace of $X$ contained in the kernel of $C$ is $\{0\}$, and the smallest $B$-invariant subspace of $X$ containing the range of $C$ is $X$, then either $T$ is one-to-one or $T$ has dense range.

Proof. Assume that $T$ has nontrivial kernel and nondense range in order to contradict the hypothesis.

Choose $x \neq 0$ in the kernel of $T$. Then $T A x=C x$, which implies either $C x \neq 0$ or $A x \in \operatorname{ker} T$. The second alternative together with (1) yield

Received by the editors November 12,1974 .

AMS (MOS) subject classifications (1970). Primary 47A15, $47 \mathrm{~B} 47$.

Key words and phrases. Invariant subspace, Lomonosov's theorem, linear operator equation. 
$T A^{2} x=C A x$, hence $C A x \neq 0$ or $A^{2} x \in$ ker $T$. We may repeat this argument indefinitely. Because the span of $\left\{A^{n} x\right\}$ cannot be an invariant subspace for $A$ contained in the kernel of $C$, there exists $y \in \operatorname{ker} T$ with $C y \neq 0$.

Since $C y=T A y$ we conclude that the kernel of $A^{*} T^{*}$ annihilates the (one-dimensional) range of $C$, or $\operatorname{ker} A^{*} T^{*} C \operatorname{ker} C^{*}$. Then $A^{*} T^{*}-T^{*} B^{*}=$ $C^{*}$ implies that $\operatorname{ker} A^{*} T^{*} \subset \operatorname{ker} T^{*} B^{*}$. For $x^{*} \in \operatorname{ker} A^{*} T^{*}$ we have $\left(A^{*} T^{*}\right) B^{*} x^{*}=A^{*}\left(T^{*} B^{*}\right) x^{*}=0$, so $\operatorname{ker} A^{*} T^{*}$ is a $B^{*}$-invariant subspace contained in the nullspace of $C^{*}$. It follows that the closure of the range of $T A$ is a $B$-invariant subspace containing the range of $C$, completing the proof of the lemma.

Begin the proof of the theorem by assuming that $A$ has no invariant subspace, $Y$ is compact, and $C=A Y-Y A$ has rank one. By one version of Lomonosov's theorem [3], there exists an operator $B$ commuting with $A$ such that $B Y g=g$ for some nonzero $g$ in $X$. Then

$$
B C=B(A Y-Y A)=A(B Y)-(B Y) A=A(B Y-I)-(B Y-I) A
$$

has rank one ( $B$ has trivial kernel since it commutes with $A$ ). Yet $B Y-I$ has nontrivial kernel and by the Fredholm alternative it has nondense range. This conclusion is contrary to the lemma.

Note added in proof. Perhaps it should be remarked that if $Y$ has rank one then the rank of $A Y-Y A$ is no greater than two. Thus the task of replacing "rank one" by "rank two" in the hypothesis is equivalent to solving the invariant subspace problem.

\section{REFERENCES}

1. V. J. Lomonosov, Invariant subspaces for operators commuting with compact operators, Funkcional. Anal. i Priložen 7 (1973), 55-56. (Russian)

2. D. G. Luenberger, Invertible solutions to the operator equation $T A-B T=$ $C$, Proc. Amer. Math. Soc. 16 (1965), 1226-1229. MR 32 \#1562.

3. H. Radjavi and P. Rosenthal, Invariant subspaces, Ergebnisse der Math., Vol. 77, Springer-Verlag, New York, 1973, p. 156.

4. J. P. Williams, On the range of a derivation, Pacific J. Math. 38 (1971), 273-279. MR $46 \# 7923$. GINIA 24595

DEPARTMENT OF MATHEMATICS, SWEET BRIAR COLLEGE, SWEET BRIAR, VIR- 